Prepared for "Conflict and Its Resolution in the Changing World of Work"

A Conference Honoring David B. Lipsky

\title{
ADVANCING DISPUTE RESOLUTION BY UNPACKING THE SOURCES OF CONFLICT: TOWARD AN INTEGRATED FRAMEWORK
}

\author{
John W. Budd \\ Center for Human Resources and Labor Studies \\ University of Minnesota \\ 3-300 Carlson School of Management \\ Minneapolis, MN 55455-0438 USA \\ jbudd@umn.edu \\ (612) 624-0357 \\ Alexander J.S. Colvin \\ ILR School \\ Cornell University \\ 454 ILR Research Building \\ Ithaca, NY 14853-3901 USA \\ ajc22@cornell.edu \\ (607) 254-8206 \\ Dionne M. Pohler \\ Centre for Industrial Relations and Human Resources \\ University of Toronto \\ 121 St. George Street \\ Toronto, ON M5S 2E8 Canada \\ dionne.pohler@utoronto.ca
}

(416) 946-3602

Version Date: September 30, 2017

\begin{abstract}
$\underline{\text { Abstract }}$
Organizational leaders, public policy makers, dispute resolution professionals, and scholars have developed diverse methods for resolving workplace conflict. But there is inadequate recognition that the effectiveness of a dispute resolution method depends on its fit with the source of a particular conflict. Consequently, it is essential to better understand where conflict comes from and how this affects dispute resolution. To these ends, this paper uniquely integrates scholarship from multiple disciplines to develop a multi-dimensional framework on the sources of conflict. This provides an important foundation for theorizing and identifying effective dispute resolution methods, which are more important than ever as the changing world of work raises new issues, conflicts, and institutions.
\end{abstract}


It isn't that they can't see the solution. It is that they can't see the problem. G.K. Chesterton (1932: 62)

Resolving disputes is both critically important and challenging. In the dynamic $21^{\text {st }}$ century world of work, myriad contracting arrangements and organizational forms, significant demographic and cultural shifts, widespread migration, interconnected global supply chains and cross-cultural teams, rapid technological change, complex webs of employment regulations (or lack thereof), and pressures for flexibility, cost-effectiveness, and competitiveness have the potential to increase conflict. Concomitantly, important theoretical and practical innovations in workplace dispute resolution have emerged in recent years (Colvin 2016; Lipsky and Avgar 2004; Lipsky, Avgar, and Lamare 2016; Roche and Teague 2011). Missing from this literature, however, is a recognition that effectively resolving a dispute requires diagnosing the sources of the conflict, and adopting a dispute resolution process that is effective for addressing this type of conflict.

Suppose a manager believes that a dispute is preventing two co-workers from working together effectively. There could be many possible reasons for this dispute. Perhaps the two workers believe that they are competing for scarce resources, such as administrative support or a single promotion opening. Maybe the workers come from different cultural backgrounds and perceive a lack of respect for each other. Perhaps one had an emotional outburst that created lingering bad feelings. It could be the case that they disagree over tasks because they foresee different uses for a product they are developing. Maybe one or both of them face difficulties communicating. Maybe all (or none) of these causes underlie this particular dispute. Not all dispute or conflict resolution methods will be equally effective in these different scenarios. For a dispute resolution method to be successful, the parties must first understand the sources of the 
conflict to choose an appropriate solution. For instance, a dispute rooted in competition for scarce resources needs to be addressed differently than a miscommunication. A clash triggered by different interpretations of information needs to be handled differently than a personality conflict. Perhaps a clarifying rule is useful in resolving a conflict over administrative support, but not when there is miscommunication. A third party intervention might usefully address disputes based on information or personalities, but only if these underlying sources of the conflict are recognized by the third party.

Significant attention has been devoted to understanding conflict across a wide variety of academic disciplines and applied fields. However, the research tends to be splintered, as scholars in various disciplines focus on particular aspects of conflict, such as resource constraints in economics, power structures in sociology, personality and emotions in psychology, and miscommunication in communication studies. While the depth that comes from disciplinary focus is highly valuable, an integrated framework is also needed to better understand, diagnose, and resolve conflict in practice (see also, Mayer 2012; Moore 2014). Zariski (2010: 219) has noted that most mediation models “continue to privilege one behavioral element and to 'bracket' or minimize the importance of the rest." We submit that this narrowness is not limited to models of mediation. Moreover, the literature tends to focus on the effects and resolution of conflict; we believe greater attention needs to be paid to the underlying sources of conflict in ways that fully reflect the breadth of factors that contribute to disputes.

We define conflict as an apparent or latent opposition between two or more parties that results from differences that are either real or imagined, and seek to uncover the diverse causes of conflict. To this end, we develop a framework in which the diverse sources of conflict are synthesized into three multi-dimensional factors or categories—structural, cognitive, and 
psychogenic (see Table 1). Structural conflicts are those that are caused by the relationship between the interests or goals of the parties. These types of conflicts are frequently seen as contests over scarce resources, but the literature on conflict and dispute resolution often fails to recognize alternative perspectives on the specific nature of interrelated interests. In addition to the structure of a situation, cognitive functioning over preferences, information, and communication can also cause conflict. Lastly, psychogenic conflict arises from the psychology of feelings or emotions: affective reactions to situations and other people that are triggered by individual personality traits or moods.

We propose that integrating these three factors and making clear their importance for explicitly identifying the various sources of conflict is an important conceptual advance in understanding conflict, which in turn can facilitate more effective dispute resolution in practice. In addition to developing this integrated framework, we also re-analyze three classic case examples found in the dispute resolution literature in order to demonstrate its unique contributions.

\section{Structural Sources of Conflict}

The interests or goals that potentially underlie a conflict can be diverse. One set of interests might involve economic resources to satisfy material needs and desires. Other goals might have a value orientation toward achieving certain outcome and procedural standards, such as fairness, inclusion, or respect. Another set of possible goals might derive from identity needs for a sense of purpose and meaning in one's life (Mayer 2012). Identity needs can be tied to group affiliations, such as racial, ethnic, or religious affinities, and particularly difficult conflicts result when group members see their collective needs for recognition, security, dignity, and flourishing threatened by other groups (Rothman 1997). For any interest or goal, structural 
conflicts are those that are caused by the relationship between the interests or goals of two or more people or organizations. We label this category "structural conflict" because the nature of these conflicts is determined by the rules, institutions, and practices in which this relationship is situated - in other words, by the structural nature of the relationship. To help understand the nature of structural conflict, we need to categorize the possible structures of these relationships. We present four different possible structures for the relationships between the interests or goals of the parties to a structural conflict.

We start with a relationship characterized by autonomous agents pursuing their selfinterests largely as equals in relationships that are relatively easy to begin and end. A classic example is a worker looking to trade their labor with an organization in a perfectly competitive labor market. When the worker's and organization's interests coincide, they should contract with each other; if either side can get a better deal with someone else, then they won't (continue to) contract with each other. A relationship between two workers might be seen in this same light when, for example, they can choose to work with each other when it serves their own interests and can otherwise choose not to without harming their careers. Owing to this focus on selfinterest, we label this an egoist structure. Conflicts that arise from this structure pertain to the terms of exchange, including following through on what is agreed to.

Alternatively, consider relationships that are expected to be more enduring. One possibility is a relationship with strongly interdependent interests in which a long-term partnership provides the greatest returns to both sides when structured appropriately. In employment relations scholarship, an employment relationship in which employers and employees have mutual interests that can be fully satisfied by both parties through well-designed, lasting human resource management policies is known as an unitarist employment relationship 
(Budd and Bhave 2010; Fox 1974). This unitarist perspective can be usefully generalized to relationships beyond the employer-employee focus of employment relations. That is, any relationship between two or more people or organizations could potentially be characterized by a unitarist structure in which long-term goals and interests are interdependent and not structurally determined to necessarily be incompatible. In this way, endemic or inherent conflicts of interest are de-emphasized or non-existent, at least in the ideal; rather, the focus is on a belief that the relationship is dominated by mutual interests that can be aligned to everyone's benefit.

There can still be conflict in such a situation because of suboptimal policies or practices, but the source of such conflict is then in these policies or practices rather than in the fundamental structure of the relationship, which has important implications for dispute resolution. Moreover, because a unitarist relationship is characterized by features that bind the parties together, that there is value in trying to maintain rather than end the relationship also has important implications for dispute resolution. Note further that unitarist relationships can often involve integrative or win-win negotiations because this can be a useful method of problem-solving focused on common interests (Walton and McKersie 1965; Fisher, Ury, and Patton 1991). But integrative negotiations could also occur in other types of relationships while integrative negotiations are not guaranteed to occur in an under-performing unitarist relationship. So a unitarist conceptualization should be seen as addressing a more fundamental issue (characterizing the structural nature of a relationship) than how to characterize a specific negotiation.

A polar alternative to a unitarist structure focusing on interest alignment is a relationship structure characterized by sharply antagonistic conflicts of interests. In order for such a relationship to exist, there needs to be some degree of interdependent interests. As such, each 
side needs each other at least to some extent, but within the relationship, there is a win-lose structure such that gains for one side come at the expense of the other. At its simplest, the classic example is a relationship largely or completely characterized by a contest over scarce resources in which one's consumption of resources limits another's ability to consume these resources. But again, because of interdependent interests there needs to be at least some element of accommodation. So the relationship can be seen as one with tensions between control or dominance and accommodation.

This tension has been extensively theorized through Marxist and other critical scholarship on the employment relationship (Edwards 1990; Thompson and McHugh 2009). This scholarship further draws attention to the extent to which inequalities in the employment relationship are embedded in diverse elements of the structural context of the employment relationship. In classical Marxism, for example, employment relationship inequality is rooted in the ownership of the means of production, which is in turn created and furthered not only by economic advantages of capital over labor, but also through social, legal, and political advantages. Relatedly, social dominance theory in social psychology draws attention to the ways in which groups seek to maintain their dominance (Sidanius and Pratto 2011). Conflict between groups, broadly defined, might therefore stem from individuals with a high social dominance orientation (Pratto et al. 1994). These insights can be applied to the consideration of all relationships in diagnosing the nature of a conflict over scarce material or non-material resources. For example, a dispute involving a temporary, contract employee and a regular full-time employee, perhaps with civil service or other protections, might involve complex power differences rooted in diverse rules, institutions, practices, identities, and values. We label relationships that are characterized 
primarily by conflicts of (broadly-defined) interests as antagonistic relationships, and recognize that the degree and sources of inequality in the relationship encompass a wide range of options.

A fourth possibility represents a mixture between the unitarist and antagonistic structures. That is, there can be longer-term relationships that have a mixture of interdependent interests in which some of the interests can be aligned (as in unitarist relationships) and some are opposed to each other (as in antagonistic relationships). Conflict is therefore mixed-motive conflict. To hold this relationship together, there needs to be some recognition of the other's opposing interests as legitimate; otherwise, it is an egoist relationship. As this type of relationship is characterized by a plurality of legitimately-recognized interests, we label this a pluralist structure. Unequal bargaining power is a key feature of a pluralist relationship and, as is also highlighted by the antagonistic model, we should fully diagnose the sources of power imbalances to understand the nature of a conflict in a pluralist structure. Mainstream U.S. employment relations scholarship embraces this pluralist structure as the most accurate representation of the contemporary employment relationship (Budd, Gomez, and Meltz 2004), but other workplace conflicts could also be situated in a pluralist structure if they are characterized by mixed-motive conflict.

In sum, one key dimension of our integrated framework on the sources of conflict is the structural context, and four key categories are instructive for considering the different structural possibilities of a relationship. Making these alternative perspectives explicit provides an important framework for understanding structural conflicts of interests which also helps actors diagnose the structural nature of a conflict, and helps them become aware of their own and others' ideologies regarding conflict. As this last point suggests, the structural nature of a conflict can be real or imagined. That is, we can think of the structural alternatives as either characterizing the actual nature of a relationship, or as what the parties imagine it to be. Indeed, 
the true structural nature of a workplace dispute might not be able to be objectively determined, in which case the key is understanding how the parties perceive the nature of their relationship while also appreciating alternative ways for thinking about the structure of a relationship. The four dimensions presented here provide the basis for doing exactly that.

\section{Cognitive Sources of Conflict}

The structural dimension emphasizes the contextual causes of conflict, but actual and potential conflicts involve people-whether as individuals, members of groups, or representatives of organizations, countries, or other entities - who act and react cognitively and emotionally. The second dimension of our framework considers factors that cause or influence conflict that relate to cognitive functioning, including interpretation, perception, information processing, decision-making, and communication. Individuals may have different preferences or differences of opinion over how to interact or solve a problem, perhaps influenced by cultural or other differences. Conflicts can arise because individuals have access or pay attention to different information, have differing or limited interpretations of the same information, or fail to

communicate effectively. By highlighting the unique features of each of these, this dimension of the framework helps diagnose how conflict often arises due to miscommunication, misperceptions, and differences of opinions over how to interact and/or solve a problem.

\section{Cognitive Frames and Preferences}

Inside and outside of the workplace, people are confronted with numerous cognitive stimuli when they witness, experience, read, or hear things. To interpret this information-rich environment and give things meaning, the human brain develops and refines a knowledge structure or interpretative schema that provides a cognitive frame for decision-making and action (Cornelissen and Werner 2014; Walsh 1995). That is, how an individual perceives and reacts to 
things they see, read, or hear depends on their cognitive frame or frame of reference such that "frames shape how individual actors see the world and perceive their own interests" (Kaplan 2008: 732; Goffman 1974). And since perception can be an important element of conflict (Rogan 2006), cognitive frames are an important potential source of conflict.

To appreciate the role of cognitive frames in influencing conflict, it is important to recognize that each individual's cognitive frame is a complex byproduct of their histories, including their culture, personal interactions, and accumulated experiences. So when organizational members have different cognitive frames, conflicts can arise because even the same problem, task, or piece of information can be interpreted differently and engender competing reactions and desired courses of action. For example, in response to an unexpected event faced by two or more parties, unique individual frames can create interpretative uncertainty "which leads to unexpected conflict about whether a relevant event occurred, how it is impacting the exchange, and/or how to respond to it" (Weber and Mayer 2014: 347). This can lead to further conflict because the parties might misinterpret the others' responses-for example, one side might perceive the other side as acting opportunistically (Weber and Mayer 2014). At an organizational level, cognitive frames shape how organizations are structured (Ranson, Hinings, and Greenwood 1980), so clashing frames can yield conflicts over the desired structural form. We can extend this to the desired structure of the employment relationship and other organizational relationships. The four structural relationship possibilities outlined earlier in the paper can be seen as cognitive frames that shape how individuals interpret these relationships and clashes over the desired relationship can be rooted in the embrace of alternative frames.

Individual frames can differ in many ways. Based on past experiences, some individuals might prefer more conservative and risk-averse behaviors. Individuals might also have differing 
expectations regarding fairness and ethics. Some might have strong beliefs about distributive justice; others about procedural justice. Some might expect high ethical standards to be followed; others might be more instrumental in their preferred approach. Money can also be interpreted through alternative frames. For example, someone who interprets their salary as an indication of their self-worth is likely to react differently than someone who sees their salary as determined by impersonal market forces.

Cultural differences can also be a source of conflict, and this can be rooted in culturallyinfluenced cognitive frames. Each individual's knowledge structure or interpretative schema develops over time as a person encounters information and organizes it. And since cognitive stimuli are experienced through the lens of one's culture, then there are important linkages between cognitive frames and culture (DiMaggio 1997). Indeed, research in cultural psychology uncovers significant cultural differences in beliefs over the importance of (a) effort or ability in determining outcomes, (b) self-enhancement motivations and thus the importance of "face", (c) the actions of others in determining one's choices, (d) conformity motivations, (e) individual dispositions or social roles for attributing behavior, and (f) holistic or analytical approaches to forecasting future events (Heine and Ruby 2010). Each of these can be thought of as a cultural difference in the way one interprets information and action, and each of these differences can lead to conflict as individuals from different cultures have different expectations and interpretations. Moreover, culture does not only potentially create conflict, it also influences how individuals react to and handle conflict (Aslani et al. 2016; Tinsley 2001). Gender differences that lead to and/or shape reactions to conflict can be thought of in a similar fashion. There are many ways, then, in which differing worldviews, expectations, and preferences can lead to conflicts in the workplace and beyond. 


\section{(Limited) Information Processing}

The human brain's limited capacity to retain information and to process it consistently can also cause or influence conflict. There are ongoing debates over how the mind actually works. Two leading theories are that the mind consists of specialized, domain-specific modules (Barrett and Kurzban 2006) or of two systems of mental processes-a fast, intuitive, heuristic system and a slower, analytical reasoning system (Evans 2008). There are complicated debates within and across these theories (Eraña 2012), but at a general level, both theories point toward a human brain that is not unitary or always internally consistent. So conflicts can arise because individuals perceive the same problem differently, such as when one uses a heuristic and another approaches it analytically. Individuals can also be motivated to process information in ways that validate preexisting beliefs, rather than by a search for accuracy (Ledgerwood, Callahan, and Chaiken 2014); again, differing levels of motivation can cause individuals to make differing interpretations and thus find themselves in conflict with each other. Indeed, dual process theories of cognition imply that individuals can have multiple reactions to the same phenomenon—such as a worker who says money isn't important when considered deliberatively, but then instinctively declines to do something that doesn't contain an extrinsic reward-which makes conflict across individuals more likely because of perceived inconsistencies in behavior. Individuals may also have different reactions to an issue depending on whether it is seen as affecting themselves or someone else, and these reactions can further be shaped by the amount of mental effort used (Paharia, Vohs, and Deshpandé 2013).

These models of cognitive limitations underlie the behavioral economics principle of bounded rationality (Brocas and Carrillo 2014). Rather than seeing individuals as extensively and carefully evaluating each situation, bounded rationality means that individuals will use 
heuristics to overcome cognitive difficulties in fully handling information processing, memory, and multi-tasking. Many of these heuristics are now well-recognized in psychology and behavioral economics, and are often labeled “cognitive biases” because they systematically appear to fall short of the decisions that would result from a careful assessment of each situation (Tversky and Kahneman 1974; Thompson and Lucas 2014). Common types of cognitive bias that result in conflict include loss aversion, anchoring, framing, fixed-pie perception, exaggeration of conflict, illusions of transparency, decision fatigue, and overconfidence. In sum, conflicts can arise because "the central characteristic of agents is not that they reason poorly but that they often act intuitively" and because "the behavior of these agents is not guided by what they are able to compute, but by what they happen to see at a given moment” (Kahneman 2003: 1469).

(In- and Out-)Group Perceptions

Cognitive processing does not occur is a social vacuum. Teams, for example, are mainstays of organizational life, and at a more fundamental level humans tend to identify with certain groups, whether on the basis of employer, occupation, race, religion, or myriad other dimensions. Social identity theory highlights the importance of group identification because humans "need to feel positive about themselves (self enhancement), and by their need to feel certain about themselves, their place in the world, and how they relate to other people (uncertainty reduction)” (Hogg 2013: 554). To derive these benefits, individuals magnify the differences between their own groups and alternative groups, emphasizing the positive aspects of their in-groups and the negative aspects of the out-groups while also acting to maintain these differences. 
This can lead to a variety of intergroup conflicts. Realistic group conflict theory emphasizes conflicts between groups over scarce resources (Sherif 1966). But social identity theory implies that intergroup conflict does not require a conflict of interest. Indeed, while overt discrimination requires intergroup interaction, other intergroup problems such as stereotyping, prejudice, stigmatizing, and implicit bias do not require interaction (Tajfel and Turner 1979). Cognitive biases can reinforce these intergroup differences, such as attributing undesirable outgroup behaviors to negative personal characteristics while negative in-group behaviors are attributed to the necessities of a particular situation (Fisher 2014).

There are many workplace applications of in-group/out-group perceptions as an important source of conflict. For example, in-group social identity attachment might cause Hispanic employees in an American workplace to speak Spanish which leads to prejudicial treatment by Whites who see them as an out-group (Bergman, Watrous-Rodriguez, and Chalkley 2008). As a second example, organizational identity can be a form of social identity, and intergroup rivalries can thus be created by corporate mergers (Terry and Amiot 2008). Because of the exaggerated differences and biases that can emerge from in-group and out-group distinctions, resolving intergroup conflict can be challenging (Halperin, Gross, and Dweck 2014; Hogg 2013), so recognizing these sources is important.

\section{(Mis)communication}

Communication transmits ideas and information between people. But there are many ways in which this can break down and thereby lead to or exacerbate conflict. Krauss and Morsella (2014) outline four paradigms for modeling how ideas and information are transferred.

In the encoding-decoding paradigm, a message is converted to a code (e.g., words), transmitted to someone else (e.g., speaking or writing), and the receiver decodes the message 
based on their understanding of what they are receiving (e.g., the meaning of the words they hear). But if the communication channel is noisy (e.g., multiple re-tellings) or if the sender and receiver have different meanings for a word (e.g., "pants” in American or British English), then misunderstandings can result, which can lead to conflict.

The intentionalist paradigm focuses on the intended meaning of a message; in this way, understanding a message is not simply a matter of decoding the actual words used, but also requires deciphering the sender's intent. Inferring this intent is done by the receiver, who filters messages through their own cognitive frame. So if there is a lack of shared understanding, the sender's intent can be misunderstood.

In the perspective-taking paradigm, the sender of a message should understand the receiver's perspective and try to phrase the message in a way that will be understood from this perspective. But similar to the intentionalist paradigm, different cognitive frames can make it difficult to understand the other's perspective, and thus it can be challenging to send a message that will be understood correctly. In short, whether one approaches communication from the perspective of the sender or the receiver, the human tendency to see the world through one's own frames, lack of understanding of alternative frames, and heightened in-group and out-group differences can lead to miscommunication. When there are multiple audiences for a message, which can be common in labor relations and other work-related settings, these communication challenges can be magnified.

In the first three perspectives, meaning lies in the message, the sender's intent, or the receiver's perspective. A fourth perspective, what Krauss and Morsella (2014) label the dialogic paradigm, identifies meaning through the communication process itself. This approach highlights shared meaning constructed in interactive communication processes, as would be common 
between co-workers, team members, supervisor-worker relationships, and labor-management negotiators. The dialogic perspective highlights the importance of active listening for creating understanding, which implies that a lack of active listening can be problematic. Interactive communication also has the potential to increase the risks of miscommunication present in the other models if back-and-forth communication magnifies rather than lessens a misunderstanding. Moreover, the interactive dimension of communication allows for the recognition that nonverbal cues and personal demeanor can shape how information is interpreted (Burgoon, Blair, and Strom 2008), which can lead to misunderstanding and conflict. Nonverbal communication that is interpreted as aggressive or that contradicts gender-based or other expectations can also cause or influence conflict (Burgoon, Guerrero, and Floyd 2010).

\section{Psychogenic Sources of Conflict}

Psychogenic conflict arises from or is affected by the psychology of feelings: affective reactions to situations and other people that are triggered by moods or individual personality traits. For example, two personalities might clash or a conflict might occur because someone is having a bad day. Psychogenic conflict should be seen as interacting with the other sources of conflict because conflict may not manifest itself if an individual does not perceive a situation, process, or outcome as threatening enough to his/her well-being or quality of life to elicit an emotional reaction (Pondy 1967). In other words, the contribution of psychogenic factors to a conflict might often be to magnify other types of differences or to escalate conflicts with structural and/or cognitive roots than to necessarily be a sole, independent source of conflict. In any case, however, the psychogenic dimension is an important potential source of conflict to identify and understand. 


\section{Emotions and Moods}

Emotions and moods are psychological experiences of feeling, or what psychologists label affective experiences. Emotions are reactions to specific causes and are therefore shortlived — such as anger, fear, or happiness. Moods, in contrast, are more of a general positive or negative feeling unrelated to a particular cause that is slightly more enduring than an emotion. The literature on conflict recognizes the importance of affective reactions that result from interpersonal conflict (Spector and Bruk-Lee 2008), but we posit that emotions and moods should also be seen as important causes and influences on conflict. That is, we focus on a causal arrow running from emotions and moods to conflict rather than the reverse.

Emotions can cause conflict through the behaviors they create or by influencing decisionmaking. Perhaps most intuitively, hard or hostile emotions such as anger, frustration, contempt, and jealousy can lead to aggressive communication behaviors such as criticism, contempt, and yelling while decreasing constructive communication behaviors such as active listening (Guerrero 2013). Humiliation (Lindner 2014) and hatred (Halperin, Gross, and Dweck 2014) are other powerful emotions that can cause (sometimes violent) conflict between individuals and also between groups. Attribution is also likely to be important; for instance, aggression is a normal response to hard emotions like anger when an individual blames someone else for intentionally causing them harm (Raver and Barling 2008). Hot emotions like fear can override self-regulation such that fight or flight reactions are automatic and reflexive (Mischel, DeSmet, and Kross 2014). Fight reactions often lead to observable manifestations of conflict while flight reactions allow conflicts to fester (Patterson et al. 2012). In these ways, emotional reactions might appear involuntary and not mediated by any form of conscious deliberation, leading to conflict; or, emotional reactions might lead to conflict when conscious deliberation causes an 
emotional reaction. Moreover, negative emotions that create a bad mood can also lead to displaced aggression in which behaviors stemming from, for example, irritability, cause a conflict with someone who was not involved in the initial affective event (Pruitt 2008). Emotional contagion can also cause (or lessen) conflict when a person's negative (positive) mood affect the moods of others (Barsade 2002).

Negative emotions and moods can also cause a conflict to escalate through the behaviors they prompt. For example, when someone finds another's negative behaviors surprising, overwhelming, and disorienting, emotional flooding can make it difficult to process information and instead focus a person's attention on reducing their negative emotions by lashing out (Guerrero 2013). Also, annoyance increases the chance of retaliation while aggression commonly creates more aggression (Pruitt 2008). It is also useful to recognize that groups can develop particularly strong emotions, as when fear or anger spreads through a crowd (Hogg 2013), so the emotional impact on conflict should not be seen in purely interpersonal terms.

In addition to possibly causing or influencing conflict through behavior, emotions and moods can lead to conflict by impacting decision-making. Individuals who are in happy or positive moods have been found to be more confident and optimistic, and make riskier decisions (George and Dane 2016). This can clash with someone who is less confident and wants to make safer decisions. The scope for conflict to arise in this way is magnified by the fact that research has found that incidental emotions "pervasively carry over from one situation to the next, affecting decisions that should, from a normative perspective, be unrelated to that emotion” (Lerner et al. 2015: 803). Discrete emotions — that is, emotions linked directly to the issue being addressed—can influence decision-making in additional ways, including the content and depth of thought, and goal activation (Lerner et al. 2015). Sometimes emotions can improve decision- 
making, and other times they can have a negative effect. But the main implication for theorizing the roots of conflict is that if decision-making is affected by an individual's emotional state, then two individuals can assess or approach the same situation differently because of different emotional states.

Personality

The second dimension of psychogenic causes or influences on conflict is personality—an individual's typical way of feeling, thinking, and behaving. One way in which personality can affect conflict is as a longer-term mood. That is, one's personality can include an overall tendency to have a positive or a negative affect such that positivity or negativity is not just a mood (state) but a more enduring trait (Barsade and Gibson 2007). So positivity or negativity as a personality trait can influence conflict in a similar way to a good or a bad mood.

Other aspects of one's personality can also cause or contribute to a conflict. A popular way to represent individual differences in personality is the five-factor model consisting of extraversion, agreeableness, conscientiousness, neuroticism, and openness. While the effect of these traits can be complex and context-dependent, individuals with high values of neuroticism and extraversion and/or low values of agreeableness may be more likely to be contentious, antagonistic, irritable, and even want to dominate others, which can be lead to behaviors that cause conflict with others (Bono et al. 2002; Sandy, Boardman, and Deutsch 2014). Similarly, those who score low on openness and conscientiousness tend to be inflexible and disorganized, which can also lead to conflict with others. At the same time, individuals who are conscientious can be more conflict prone if they are excessively fastidious while open individuals can be argumentative and not afraid of conflict (Bono et al. 2002). We assert that the potential importance of personality traits for causing conflict is magnified when we appreciate the 
prospect of personality differences across individuals. So it's not just that a certain personality type might be more conflict prone, it's also that different personality types might clash to create conflict—such as people who are low on conscientiousness interacting with those who are high on that same dimension. This is reinforced by research on the relationship between group composition and performance (Mohammed and Angell 2003).

Personality can also affect conflict by affecting an individual's attributions such that different personality types have a tendency to see a conflict as either task- or relationship-based, and the influence of personality on this tendency is stronger when one accounts for the personality of both people involved (Bono et al. 2002). For example, differences in levels of extraversion leads to more conflict that is seen as task-based whereas conscientiousness is associated with relationship-based attributions of the nature of a conflict.

\section{Managing Conflict at its Sources}

Disputes can be resolved in various ways. Many conflicts that arise in interactions between individuals and groups are resolved through established processes, institutions, and/or rules to which people acquiesce or follow. For instance, the boss gets the final say about raises or promotions when two co-workers are competing for the same job, and a law may specify obligations for union negotiations with an organization. When rules and institutions are effective, some conflicts never manifest in a material or observable way, and other conflicts are channeled into and resolved in socially-acceptable forums. However, when this form of conflict resolution fails due to structural, cognitive, and/or psychogenic reasons, different dispute resolution methods are needed.

Negotiation between two or more parties is another common method of conflict resolution. Negotiation may occur over rules and processes, as well as distributive outcomes. 
Making negotiations more complex, many situations involve a mixture of distributive and integrative issues and the effective negotiator must try to balance achieving successful resolution of both types of issues at once.

When established rules and institutions or negotiations fail, sometimes third-party assistance is required. Third parties can provide a range of different types of assistance to the key actors experiencing the conflict. Some mechanisms, such as arbitration, involve a third party deciding what the outcome of the conflict will be. Other mechanisms, such as mediation, involve a third party assisting the key actors in reaching a negotiated resolution of the conflict.

These dispute resolutions methods are well-known (Coleman, Deutsch, and Marcus 2014; Lipsky, Avgar, and Lamare 2016; Roche, Teague, and Colvin 2014) and do not need to be presented in further detail here. But there is a need to better integrate a comprehensive framework on the causes of conflict with different approaches to dispute resolution- that is, to manage conflict at its sources. Conceptually, it is important for the dispute resolution literature to identify the aspects of conflict that different methods are more or less better equipped to address to set a foundation for future empirical work analyzing these links. And practically this is important for helping dispute resolution professionals and others identify appropriate and effective methods for resolving a particular dispute. In this section, we develop a number of ways in which conflict resolution can be more closely tied to its root causes. Given the sometimes-complex interactions among different factors in a conflict, we cannot fully develop all of the implications, and there is scope for much additional work.

\section{Resolving Structural Conflict}

Resolving structural conflicts requires diagnosing whether a particular conflict is best characterized by the egoist, unitarist, pluralist, or antagonistic mindsets. If the structure of a 
relationship is characterized by unitarism, then the structure likely is not the problem. Rather, conflict may stem from failing to recognize the mutual nature of the relationship which implies that the frames of the participants need to change, and/or that the particular policies and practices need revising to reflect the unitarist nature of the relationship. In contrast, if the structure of a relationship is better characterized by an egoist structure, then resolving a conflict may require determining whether there are realistic alternatives to the proposed exchange, and ensuring each side fulfills its side of the bargain. In a pluralist relationship, more attention needs to be paid to the relationship and hence the other's interests because there are fewer alternatives so the parties need to live with the consequences to a greater extent than in an egoist relationship. Failing to recognize these differences will make it more difficult to effectively address conflict rooted in different structural contexts.

Recognizing alternative structural forms of conflict is also important for appropriately factoring in issues of power. In an egoist relationship, power is less important than self-interest. If someone gives you a good deal, take it; if not, take your next best alternative. In a unitarist relationship, a focus on power likely interferes with finding interest-aligning policies. In contrast, power differences are likely a significant aspect of an antagonistic relationship, and distributive negotiations would be fully consistent in this structure. Integrative bargaining is very difficult in an antagonistic structure. In a pluralist relationship, distributive and integrative negotiations are likely, and the parties or third party dispute resolution actors would likely need to ensure that power is not exercised in an overly aggressive way that undermines the shared interests and enduring nature of the relationship.

The appropriate roles of rules and third party interventions for resolving conflict will also vary with the nature of the relationship. In an egoist relationship, the existence of rules governing 
exchanges should serve to prevent conflict by allowing efficient exchanges to occur. Third party intervention would only be necessary to resolve conflict to the degree that enforcement of these rules is necessary, including adherence to agreed-upon terms, suggesting arbitration-type procedures that allow adjudication of rule violations. In an unitarist relationship, the mutuality of the situation suggests that rules are not necessary to police behaviors, nor is arbitration of conflicts desirable. Rather mediation-type third party interventions are most useful in helping the parties to recognize their mutual interests and resolve any barriers to achieving the integrative potential inherent in their relationship. However, if the relationship is antagonistic in structure, efforts to mediate in search of common interests risk obscuring the fundamental oppositions of interest that drive conflict in the relationship. Rules and arbitration-type third party interventions may be useful, but only to the extent that they are directed at addressing the antagonistic structure of the relationship and correcting the power imbalances that are inherent in it. By contrast, pluralist relationships are most open to a range of responses, including establishing governing rules and both mediation- and arbitration-type third party interventions, reflecting the diverse nature of distribution and integrative issues inherent in this type of relationship.

\section{Resolving Cognitive Conflict}

Diagnosing different factors that cause or influence conflict that relate to cognitive functioning, including interpretation, perception, information processing, decision-making, and communication, is also important for applying effective approaches to dispute resolution. When a conflict is rooted in differing cognitive frames, then there are various techniques to address these differences. Frame alignment processes include frame bridging, amplification, extension, and transformation (Snow et al. 1986). An alternative way to approach this is via a sequence of unfreezing, changing, and refreezing cognitive frames (Barr, Stimpert, and Huff 1992). If one 
party to a dispute recognizes that a conflict is rooted in conflicting frames, that party can try to align the frames (Cornelissen and Werner 2014), and this can also be an important mediator task (Bodtker and Jameson 1997).

One aspect of frame adjustment to resolve a dispute is reframing the structural nature of a conflict (Mayer 2012). For instance, integrative bargaining can be very difficult if the parties believe that the nature of their relationship is defined by an antagonistic structure. Reorienting this mindset by changing the parties' cognitive frame about the structural nature of the relationship is likely needed if integrative bargaining is to be successful. But to recognize the fundamental importance of cognitive frames is to appreciate that cognitive frames are not simply lenses for viewing the structural nature of a particular conflict; rather, they are potentially a cause of conflict by shaping how participants interpret information and communication. So exaggerated differences between in-group and out-group members can be occurring because of a cognitive frame. Interventions can be constructed to change this cognitive frame and thereby open up possibilities for conflict resolution (Halperin, Gross, and Dweck 2014). More generally, interactional frame theories assert that a key aspect of any conflict is the process of the parties co-creating not only the meaning of the issues, but the meaning of their relationship, and alignment needs to occur to resolve a dispute (Dewulf et al. 2009).

Turning to conflict that has an aspect of limited information processing, people can more easily identify cognitive errors made by others than themselves (Pronin 2007). Providing individuals training in decision-making biases and teaching them critical thinking and selfawareness can help them become aware of decision-making blind spots to work through this type of cognitive conflict. A complementary approach is to consider and shape the participants' motives for information processing - especially enhancing an accuracy motivation, lessening a 
defense motivation, and managing an impression motivation (Ledgerwood, Callahan, and Chaiken 2014). Rules and choice architecture can also be used to guide individuals around undesirable heuristics and cognitive biases. Communication strategies for overcoming misinformation have also been developed (Lewandowsky et al. 2012).

Recognizing when miscommunication causes or contributes to a conflict also points to specific conflict resolution strategies. For example, the four paradigms of communication outlined earlier yield a number of principles, including avoiding communication channels with low signal-to-noise ratios, listening for the intended meanings of what's being said, communicate in ways that the listener will understand your intent and that reflects the listener's perspective, and establishing conditions in which an effective dialogue can occur (Krauss and Morsella 2014). Strategies to address communication challenges in cross-cultural interactions are particularly important yet challenging (van Meurs and Spencer-Oatey 2007).

Lastly, the strengths and weakness of both negotiation and different forms of third party assistance relate to solving cognitive conflict. Negotiation may more easily resolve access to information, whereas neutral outside third parties such as mediators may be required to help resolve conflict due to different frames of reference held by the parties or difficulties in communicating (Zariski 2010). Use of rules that structure interactions and arbitration-type third party interventions that impose a solution on the parties may provide ways of resolving an immediate dispute, however these approaches may be less effective in preventing recurrence of conflict if the roots of that conflict are cognitive, since they do not directly address the key sources. 


\section{Resolving Psychogenic Conflict}

Psychogenic conflict is perhaps the most difficult type of conflict to tackle. This aspect of conflict is not easily resolved through negotiation, nor is it likely to be truly resolved by the imposition of a solution by a third party such as a manager or an arbitrator. Rules and choice architecture may prevent stressful situations from resulting in emotional flare-ups (Lerner et al. 2015). But the most accessible strategy is to give people tools to work through their own emotions, or to control their moods in different situations, either in advance of a conflict or during it. When dealing with hot emotions, cooling strategies such as taking a time-out or a break and trying to re-orient an individual's attention to be more reflective and self-distanced rather than self-immersed can facilitate problem solving (Mischel, DeSmet, and Kross 2014; Lerner et al. 2015).

If anger (Pruitt 2008) or humiliation (Lindner 2014) are contributing to a conflict, then the other party can potentially lessen these emotions by acknowledging them. More generally, cycles of negativity can be broken by positive behavior by others, especially when the extent of the positive behavior exceeds expectations (Guerrero 2013). It is also useful to recognize that some emotions can be helpful in trying to resolve a conflict to the extent that individuals want to feel a sense of belonging and recognition (Lindner 2014). With respect to personality, an understanding of how different personality types approach not only conflict, but feeling, thinking, and behavior more generally can be useful to understand how to best engage an individual constructively and productively. Mediators and other facilitators who recognize the

psychogenic aspects of conflict can be prepared to intervene in productive ways, but this is unlikely to happen if the psychogenic elements of a conflict are unrecognized. 
Transformative mediation is an approach to third party intervention that more directly focuses on psychogenic conflict (Bush and Folger 1994). In transformative mediation, the mediator seeks to improve the relationship between the parties and empower the parties so that they will be able to resolve future conflicts themselves. Part of the premise of transformative mediation is that there are psychogenic aspects to conflict that become embedded in relationships between individuals and that it is futile to hope to achieve long-term resolution of the conflict until these negative psychogenic aspects of the relationship are addressed.

\section{Applications of the Framework}

An important test of the usefulness of the framework we have set out here is whether it enhances our ability to analyze episodes of conflict and to identify effective conflict resolution approaches. As an initial effort to test whether our framework is useful in this way, in this section we employ it in re-analyzing three classic examples from the conflict resolution literature. The examples we draw on are central illustrative cases described in three seminal works: James Kuhn’s 1961 book Bargaining in Grievance Settlement; William Ury, Jeanne Brett, and Stephen Goldberg’s 1989 book Getting Disputes Resolved: Designing Systems to Cut the Costs of Conflict; and Karen Jehn’s 1997 article “A Qualitative Analysis of Conflict Types and Dimensions in Groups.” Each of these works adopts case examples to develop frameworks, theories, and analyses of how conflict and conflict resolution work. Our intent is not to criticize any of these authors' frameworks or insights, all of which we view as important advances in the field of dispute resolution. Rather our aim is to show how our framework provides additional insights that build on existing work, which furthers our understanding of the sources of conflict and thus aids its effective resolution. 
We begin with Kuhn’s Bargaining in Grievance Settlement. At the heart of Kuhn’s book investigating grievance processes is the classic example he describes of the "hot tread" grievance (Kuhn 1961: 58-77). On the surface, the hot tread grievance is a story about a grievance filed by workers in a plant making tires for earthmovers who alleged that they had suffered injuries from having to handle overly hot tire treads, leading to burns, and costing them compensation under the plant's piecework pay scheme. The grievance proceeded to arbitration, where the arbitrator denied the union's grievance. Kuhn shows how a much more complex set of interactions, representing the "Real 'Hot Tread' Case," lay underneath this formal grievance and arbitration process. The real hot tread case involved concerns by the workers that they would not be able to make their usual earnings on a mixture of piecework and hourly pay. The union's committeeman who represented them was unable to negotiate a resolution of these concerns with the foreman. Due to a combination of the committeeman's lack of experience and political support and the increasing aggravation of the workers, the conflict intensified and the workers decided to exert their power on the shopfloor by filing a series of grievances. After a period of heightened conflict, union and management representatives were able to negotiate a resolution that dealt with pay concerns and removed some outstanding grievances. Labor relations on the shopfloor then returned to a more cooperative pattern.

Kuhn uses the hot tread case to illustrate the process of fractional bargaining, where work groups exert their power on the shopfloor to negotiate resolution of conflicts by going around the formal grievance procedure. Applying our framework to this case more clearly reveals the underlying structural, cognitive, and psychogenic elements of the hot tread conflict that each need to be recognized to produce an effective resolution. 
The structural elements of the hot tread case are in some ways the most obvious. The grievance that went to arbitration is a classic example of opposing interests that are resolved through an adversarial adjudicative system. From either an antagonistic or pluralist perspective, there was a structural conflict of interest that produced an antagonistic relationship; management was trying to maintain production while the employees were trying to ensure they received adequate compensation. Yet at the same time, the real hot tread case shows how cognitive framing can be an underlying source of conflict. The reason that the workers were so upset about the problems with the machine was that it interfered with their ability to "make out" their expected earnings - that is, their cognitive frame for understanding the wage-effort bargain included expected daily earnings. When the combination of reduced piecework pay and inadequate supplemental hourly pay meant that they were unable to earn their expected wage, this was perceived as a violation of the normative expectations embedded within their cognitive frame, thereby producing the true conflict of the real hot tread case.

In addition to its structural and cognitive elements, the hot tread case also illustrates the psychogenic sources of conflict. Although hot treads were a frequent enough occurrence that the plant provided workers with asbestos pads to protect their hands while handling the tires, in this particular case they had been treated at the hospital, producing a shift in emotions and moods. Here is how one of their own union leaders viewed the situation:

After talking to the committeeman and crew, he was not convinced that the men had received any serious burns, though they undoubtedly had been inconvenienced. Explaining his stand later, he said that if the men had not been in bad shape when they left for the hospital, "they sure were convinced of it when they came back. The nurse was pretty dumb and had put Unguentine on and bandaged their hands in yards of gauze. They were pretty worked up after that" (Kuhn 1961: 63, emphasis added). 
An important element in the hot tread case is the layering of physical injury and emotional reaction to it, which produced an intensified psychogenic conflict apart from the structural issues of what the rule was for compensation when there were problems with the machinery or conflicts arising from clashing cognitive frames about what was necessary to "make out" their day's earnings. To fully resolve the conflicts in this case requires understanding all of these layers.

Our second case example is taken from Ury, Brett, and Goldberg's Getting Disputes Resolved, which presents their framing of rights, power, and interests as three approaches to resolving disputes. They illustrate this framework with the classic case of a dispute involving a miner's stolen boots (Ury, Brett, and Goldberg 1989: 3-10). The miners, who owned their own safety boots, left their work clothes and boots at the mine between shifts. One night a miner arrived to discover his boots were gone and complained to the shift boss that they had been stolen, meaning that he was unable to work without safety boots and would lose a shift's pay. Ury, Brett, and Goldberg describe the shift boss's initial denial of the company's responsibility to replace the lost boots as an example of a rights-based attempt to resolve the dispute based on the rules. The miner responded to this denial of his complaint by organizing a wildcat strike among his fellow miners. Ury, Brett, and Goldberg describe this as a power-based method of trying to resolve the dispute. Finally, the superintendent of the mine stepped in and proposed replacing the stolen boots so the miners could get back to work, the miner who lost his boots would earn his shift pay, and the mine would not suffer the costs of lost production. The authors describe this last approach as an interest-based method of resolving the dispute.

The framing of interests, rights, and power, is a very valuable way of thinking about different processes for resolving disputes. But it is less successful in revealing the underlying sources or nature of the conflict processes that drive disputes. At the outset, the mining dispute 
arose from a conflict in cognitive frames: the miner's idea of fairness meant he believed that he should not lose a shift's pay due to someone stealing his boots versus the shift boss's idea that boots were personal property and not the company's responsibility. Layered on top of this conflict in cognitive frames over what was considered "fair" in this situation, however, were psychogenic elements of the emotions expressed in this interaction. Consider how Ury, Brett, and Goldberg describe the way in which each party communicate their respective positions:

"Hard luck!" the shift boss responded. "The company isn't responsible for personal property left on company premises. Read the mine regulations!"

The miner grumbled to himself, "I'll show them! If I can't work this shift, neither will anyone else!” (Ury, Brett, and Goldberg 1989: 3, emphasis added).

These comments show invective language, exclamations, and passionate responses to having a request denied. As the mood escalates from a discussion of a problem to a clash of personalities in the workplace, we see the conflict becoming increasingly psychogenic in nature, leading to the expression of the wildcat strike.

Finally, we see the superintendent returning to the structural conflicts underlying this dispute. Why do the different cognitive frames and psychogenic conflicts matter so much here? The reason is that there was also a structural conflict between the mine management's interest in maintaining its authority and ensuring production at the mine and the workers' interests in maintaining their earnings from working their shifts. While there is an antagonistic element to this relationship, it is resolved in pluralistic fashion as the superintendent recognizes the mutual interest involved in getting work resumed so that production can be maintained and the workers continue to earn their pay. But this structural resolution is quite possibly a temporary solution because the cognitive and psychogenic aspects have not been addressed and thus may keep festering until another flashpoint again brings them to the surface. 
Our third illustration is drawn from Karen Jehn's seminal article on intragroup conflict, “A Qualitative Analysis of Conflict Types and Dimensions in Organizational Groups” (Jehn 1997). Jehn uses qualitative evidence from a study of six work groups at a household-goods moving company to develop a model of the nature and effect of intragroup conflict. At the core of Jehn's model is the typology of three different types of conflict: task conflict, involving disagreements over "the content and goals of the work"; relationship conflict, involving "interpersonal relationships"; and process conflict, involving "how tasks would be accomplished” (Jehn 1997: 551). In this model, the impact of these three types of conflict on group performance is moderated by other characteristics of the conflict: resolution potential, acceptability norms, emotionality, and importance.

Our own analysis of three sources of conflict, structural, cognitive, and psychogenic, should not be seen as an alternative schema to Jehn's three types of conflict, which is a categorization focusing on the locus of conflict. Rather, our framework represents an analysis of the underlying sources of conflict that can produce manifestations in the three types of conflict that Jehn identifies. We illustrate this point by examining some of the examples of conflicts that Jehn uses to illustrate her model. For example, she illustrates task conflict with the following interview comments and field notes:

"Sometimes people get irritated at each other about work matters..."

"We usually fight about work things - interpreting our reports, disagreeing about government regulations."

"The pace is so fast. They don't have time to deliberate so that it's a constant give and take. It's very busy and they are all doing ten things at once yet the need to reach agreement on the border decision” (Jehn 1997: 542, emphasis added).

These are three good examples of situations where the locus of conflict is the task. But they each have a different source. The first illustrates psychogenic conflict where a mood or emotion (i.e., irritability) was driving the conflict. The second is an example of cognitive conflict where the 
individuals had different interpretative schema over how to interpret the reports and government regulations. The third shows structural sources of conflict where the pace of work and the tension between organizational demands and individual work capacity produces conflict.

Jehn also gives examples of relationship and process conflicts with contrasting sources. Descriptions by an informant of a relationship conflict with a co-worker that stated "Her attitude just stinks" and "I just can’t stand her attitude and her voice” (Jehn 1997: 542) suggest a conflict rooted in psychogenic reactions of emotion and mood that may be further rooted in personality differences, whereas other relationship conflict might be rooted in cognitive elements such as cultural differences or miscommunication. Similarly, the following description of a process conflict suggests structural roots to conflict based on organizational incentives: "The group was discussing which operations would include Pat....'I'm not sure if it's his responsibility to be included in this. He doesn't count on our budget." (Jehn 1997: 542). And yet other process conflicts are rooted in different cognitive perceptions of the most efficient way to get things done: "Jeff suggested that he finish the mail so Joan could go on break but Mary told him that he wouldn't be fast enough. They were having a big problem figuring out how to utilize their people and schedule breaks efficiently” (Jehn 1997: 542).

Our point in re-analyzing these examples is not to argue against the usefulness of the frameworks and insights developed by Kuhn (1961), Ury, Brett, and Goldberg (1989), Jehn (1997), or others. Rather our point is that the examples presented in each of these three classic works examining conflict and conflict resolution illustrate the need for additional treatments on the underlying structural, cognitive, and psychogenic sources of conflict. By incorporating an analysis of the underlying sources of conflict into our thinking about conflict we can improve our 
understanding of what is really driving the conflicts we observe and as a result, develop better approaches to resolving them.

\section{Conclusion}

Conflict is an enduring feature of all social life. In the workplace, conflict often manifests in disputes between co-workers, collective agreement negotiations between managers and employees over wages and workloads, or any one of an infinite number of interactions that may occur between various organizational stakeholders. A tremendous amount of thought and research has been put into understanding conflict across a wide variety of academic disciplines, including but not limited to industrial and employment relations, management, psychology, sociology, political science, economics, communications, and law. This has generated frameworks and theories that have been applied in a number of practical settings. While it is not possible to be completely comprehensive, we attempt to incorporate key insights from many of these literatures into our framework.

Our paper both synthesizes and integrates diverse multidisciplinary views on conflict to create a unique framework for understanding major sources of conflict—distilled down to three multi-dimensional factors or categories - structural, cognitive, and psychogenic. Disputes can be multi-faceted with numerous causes that interact in complex ways. We assert that it is important to conceptually distinguish different aspects of the full range of sources of conflict to appreciate the nature of each particular dispute, because what can be considered "effective" dispute resolution must be rooted in a comprehensive and accurate understanding of its origins. Our approach is not intended to imply that each multi-dimensional factor is independent or can be isolated in practice. But by identifying the separate pieces of a potentially complex puzzle into an 
integrative framework, researchers and practitioners are better equipped to understand, analyze, diagnose, and propose solutions to these complex puzzles.

While many of the sources we incorporate and factors we highlight are already either implicitly or explicitly recognized in the conflict and dispute resolution literatures, the focus is often on using these concepts to resolve a dispute and understand the effects of conflict-for example, affective reactions to conflict and affective barriers to dispute resolution. We are ultimately calling for more explicit attention to be devoted to the causes of conflict, and we provide a contribution toward this end that adopts a breadth equal to the complexity of many conflicts, rather than privileging particular aspects.

Practically, we believe that our resulting framework helps generate new ideas for developing more appropriate and effective dispute resolution methods that are solidly based in a renewed understanding of a robust range of sources of conflict. We illustrate the added contribution of our framework vis-à-vis seminal approaches in the field of dispute resolution by re-analyzing classic case examples. Our framework highlights additional considerations for understanding the sources of conflict in each of these cases, and the potential for the (in)effective resolution of conflict that may result from an (in)accurate diagnosing of the underlying sources. 


\section{References}

Aslani, Soroush, et al. (2016) "Dignity, Face, and Honor Cultures: A Study of Negotiation Strategy and Outcomes in Three Cultures,” Journal of Organizational Behavior 37(8): 1178-201.

Barr, Pamela S., John L. Stimpert, and Anne S. Huff (1992) "Cognitive Change, Strategic Action, and Organizational Renewal,” Strategic Management Journal 13(S1): 15-36.

Barrett, H. Clark, and Robert Kurzban (2006) “Modularity in Cognition: Framing the Debate,” Psychological Review 113(3): 628-47.

Barsade, Sigal G. (2002) "The Ripple Effect: Emotional Contagion and its Influence on Group Behavior,” Administrative Science Quarterly 47(4): 644-75.

Barsade, Sigal G., and Donald E. Gibson (2007) "Why Does Affect Matter in Organizations?” Academy of Management Perspectives 21(1): 36-59.

Bergman, Mindy E., Kristen M. Watrous-Rodriguez, and Katherine M. Chalkley (2008) "Identity and Language: Contributions to and Consequences of Speaking Spanish in the Workplace,” Hispanic Journal of Behavioral Sciences 30 (1): 40-68.

Bodtker, Andrea M., and Jessica K. Jameson (1997) "Mediation as Mutual Influence: Reexamining the Use of Framing and Reframing," Conflict Resolution Quarterly 14(3): 237-49.

Bono, Joyce E., Terry L. Boles, Timothy A. Judge, and Kristy J. Lauver (2002) “The Role of Personality in Task and Relationship Conflict,” Journal of Personality 70(3): 311-44.

Brocas, Isabelle, and Juan D. Carrillo (2014) "Dual-Process Theories of Decision-Making: A Selective Survey,” Journal of Economic Psychology 41: 45-54.

Budd, John W., and Devasheesh Bhave (2010) “The Employment Relationship.” In Adrian Wilkinson et al. (eds.), Sage Handbook of Human Resource Management (London: Sage): 51-70.

Budd, John W., Rafael Gomez, and Noah M. Meltz (2004) "Why a Balance is Best: The Pluralist Industrial Relations Paradigm of Balancing Competing Interests.” In Bruce E. Kaufman (ed.), Theoretical Perspectives on Work and the Employment Relationship (Champaign, IL: Industrial Relations Research Association): pp. 195-227.

Burgoon, Judee K., J. Pete Blair, and Renee E. Strom (2008) “Cognitive Biases and Nonverbal Cue Availability in Detecting Deception,” Human Communication Research 34(4): 57299. 
Burgoon, Judee K., Laura K. Guerrero, and Kory Floyd (2010) Nonverbal Communication (New York: Routledge).

Bush, Robert A.B., and Joseph P. Folger. (1994) The Promise of Mediation: Responding to Conflict Through Empowerment and Recognition. (San Francisco, CA: Jossey-Bass).

Chesterton, G. K. (1932) “The Point of a Pin,” Saturday Evening Post, Vol. 205, Issue 12 (September 17): 6+.

Coleman, Peter T., Morton Deutsch, and Eric C. Marcus, eds. (2014) The Handbook of Conflict Resolution: Theory and Practice, 3rd ed. (San Francisco: Jossey-Bass).

Colvin, Alexander J.S. (2016) "Conflict and Employment Relations in the Individual Rights Era.” In David B. Lipsky, Ariel C. Avgar, and J. Ryan Lamare (eds.), Managing and Resolving Workplace Conflict-Advances in Industrial and Labor Relations, Volume 22 (Bingley, West Yorkshire: Emerald Group Publishing): 1-30.

Cornelissen, Joep P., and Mirjam D. Werner (2014) "Putting Framing in Perspective: A Review of Framing and Frame Analysis Across the Management and Organizational Literature,” Academy of Management Annals 8(1): 181-235.

Dewulf, Art, et al. (2009) "Disentangling Approaches to Framing in Conflict and Negotiation Research: A Meta-Paradigmatic Perspective,” Human Relations 62(2): 155-93.

DiMaggio, Paul (1997) “Culture and Cognition,” Annual Review of Sociology 23: 263-87.

Edwards, P.K. (1990) "Understanding Conflict in the Labour Process: The Logic and Autonomy of Struggle.” In David Knights and Hugh Willmott (eds.), Labour Process Theory (London: Palgrave Macmillan): 125-52.

Eraña, Angeles (2012) "Dual Process Theories Versus Massive Modularity Hypotheses," Philosophical Psychology 25(6): 855-72.

Evans, Jonathan St. B.T. (2008) "Dual-Processing Accounts of Reasoning, Judgment, and Social Cognition,” Annual Review of Psychology 59: 255-78.

Fisher, Roger, William Ury, and Bruce Patton (1991) Getting to YES: Negotiating Agreement Without Giving In, 2nd ed. (New York: Penguin Books).

Fisher, Ronald J. (2014) “Intergroup Conflict.” In Peter T. Coleman, Morton Deutsch, and Eric C. Marcus (eds.), The Handbook of Conflict Resolution: Theory and Practice, 3rd ed. (San Francisco: Jossey-Bass): 230-52.

Fox, Alan (1974) Beyond Contract: Work, Power and Trust Relations (London: Farber and Farber). 
George, Jennifer M., and Erik Dane (2016) “Affect, Emotion, and Decision Making," Organizational Behavior and Human Decision Processes 136: 47-55.

Goffman, Erving (1974) Frame Analysis: An Essay on the Organization of Experience (Cambridge, MA: Harvard University Press).

Guerrero, Laura K. (2013) “Emotion and Communication in Conflict Interaction.” In John G. Oetzel and Stella Ting-Toomey (eds.), The Sage Handbook of Conflict Communication (Thousand Oaks, CA: Sage): 105-32.

Halperin, Eran, James J. Gross, and Carol S. Dweck (2014) "Resolving Intractable Intergroup Conflicts: The Role of Implicit Theories about Groups.” In Peter T. Coleman, Morton Deutsch, and Eric C. Marcus (eds.), The Handbook of Conflict Resolution: Theory and Practice, 3rd ed. (San Francisco: Jossey-Bass): 384-99.

Heine, Steven J., and Matthew B. Ruby (2010) “Cultural Psychology,” Wiley Interdisciplinary Reviews: Cognitive Science 1(2): 254-66.

Hogg, Michael A. (2013) "Intergroup Relations.” In John DeLamater and Amanda Ward (eds.), Handbook of Social Psychology, 2nd ed. (New York: Springer): 533-61.

Jehn, Karen A. (1997) "A Qualitative Analysis of Conflict Types and Dimensions in Organizational Groups,” Administrative Science Quarterly 42(3): 530-57

Kahneman, Daniel (2003) "Maps of Bounded Rationality: Psychology for Behavioral Economics," American Economic Review 93(5): 1449-75.

Kaplan, Sarah (2008) "Framing Contests: Strategy Making under Uncertainty," Organization Science 19(5): 729-52.

Krauss, Robert M., and Ezequiel Morsella (2014) “Communication and Conflict.” In Peter T. Coleman, Morton Deutsch, and Eric C. Marcus (eds.), The Handbook of Conflict Resolution: Theory and Practice, 3rd ed. (San Francisco: Jossey-Bass): 168-81.

Kuhn, James W. (1961) Bargaining in Grievance Settlement: The Power of Industrial Work Groups (New York: Columbia University Press).

Ledgerwood, Alison, Shannon P. Callahan, and Shelly Chaiken (2014) "Changing Minds: Persuasion in Negotiations and Conflict Resolution.” In Peter T. Coleman, Morton Deutsch, and Eric C. Marcus (eds.), The Handbook of Conflict Resolution: Theory and Practice, 3rd ed. (San Francisco: Jossey-Bass): 533-57.

Lerner, Jennifer S., Ye Li, Piercarlo Valdesolo, and Karim S. Kassamet (2015) "Emotion and Decision Making,” Annual Review of Psychology 66: 799-823. 
Lewandowsky, Stephan, Ullrich K.H. Ecker, Colleen M. Seifert, Norbert Schwarz, and John Cook (2012) "Misinformation and its Correction: Continued Influence and Successful Debiasing,” Psychological Science in the Public Interest 13(3): 106-31.

Lindner, Evelin G. (2014) "Emotion and Conflict: Why It Is Important to Understand How Emotions Affect Conflict and How Conflict Affects Emotions.” In Peter T. Coleman, Morton Deutsch, and Eric C. Marcus (eds.), The Handbook of Conflict Resolution: Theory and Practice, 3rd ed. (San Francisco: Jossey-Bass): 283-309.

Lipsky, David B., and Ariel C. Avgar (2004) "Commentary: Research on Employment Dispute Resolution: Toward a New Paradigm,” Conflict Resolution Quarterly 22(1-2): 175-89.

Lipsky, David B., Ariel C. Avgar, and J. Ryan Lamare, eds. (2016) Managing and Resolving Workplace Conflict--Advances in Industrial and Labor Relations, Volume 22 (Bingley, West Yorkshire: Emerald Group Publishing).

Mayer, Bernard (2012) The Dynamics of Conflict: A Guide to Engagement and Intervention, 2nd ed. (San Francisco: Jossey-Bass).

Mischel, Walter, Aaron L. DeSmet, and Ethan Kross (2014) "Self-Regulation in the Service of Conflict Resolution.” In Peter T. Coleman, Morton Deutsch, and Eric C. Marcus (eds.), The Handbook of Conflict Resolution: Theory and Practice, 3rd ed. (San Francisco: Jossey-Bass): 310-30.

Mohammed, Susan, and Linda C. Angell (2003) "Personality Heterogeneity in Teams: Which Differences Make a Difference for Team Performance?” Small Group Research 34(6): 651-77.

Moore, Christopher W. (2014) The Mediation Process: Practical Strategies for Resolving Conflict, 4th ed. (San Francisco: Jossey-Bass).

Paharia, Neeru, Kathleen D. Vohs, and Rohit Deshpandé (2013) “Sweatshop Labor is Wrong Unless the Shoes are Cute: Cognition Can Both Help and Hurt Moral Motivated Reasoning,” Organizational Behavior and Human Decision Processes 121 (1): 81-88.

Patterson, Kerry, et al. (2012) Crucial Conversations: Tools for Talking When Stakes Are High, 2nd ed. (New York: McGraw-Hill).

Pondy, Louis R. (1967) “Organizational Conflict: Concepts and Models,” Administrative Science Quarterly 12(2): 296-320.

Pratto, Felicia, et al. (1994) "Social Dominance Orientation: A Personality Variable Predicting Social and Political Attitudes,” Journal of Personality and Social Psychology 67(4): 74163. 
Pronin, Emily (2007) "Perception and Misperception of Bias in Human Judgment," Trends in Cognitive Sciences 11(1): 37-43.

Pruitt, Dean G. (2008) “Conflict Escalation in Organizations.” In Carsten K.W. De Dreu and Michele J. Gelfand (eds.), The Psychology of Conflict and Conflict Management in Organizations (New York: Lawrence Erlbaum Associates): 245-66.

Ranson, Stewart, Bob Hinings, and Royston Greenwood (1980) "The Structuring of Organizational Structures,” Administrative Science Quarterly 25(1): 1-17.

Raver, Jana L., and Julian Barling (2008) "Workplace Aggression and Conflict: Constructs, Commonalities, and Challenges for Future Inquiry.” In Carsten K.W. De Dreu and Michele J. Gelfand (eds.), The Psychology of Conflict and Conflict Management in Organizations (New York: Lawrence Erlbaum Associates): 211-44.

Roche, William K., and Paul Teague (2011) "Firms and Innovative Conflict Management Systems in Ireland,” British Journal of Industrial Relations 49(3): 436-59.

Roche, William K., Paul Teague, and Alexander J.S. Colvin, eds. (2014) The Oxford Handbook of Conflict Management in Organizations (Oxford: Oxford University Press).

Rogan, Randall G. (2006) “Conflict Framing Categories Revisited,” Communication Quarterly 54(2): 157-73.

Rothman, Jay (1997) Resolving Identity-Based Conflict in Nations, Organizations, and Communities (San Francisco: Jossey-Bass).

Sandy, Sandra V., Susan K. Boardman, and Morton Deutsch (2014) "Personality and Conflict." In Peter T. Coleman, Morton Deutsch, and Eric C. Marcus (eds.), The Handbook of Conflict Resolution: Theory and Practice, 3rd ed. (San Francisco: Jossey-Bass): 400-29.

Sherif, Muzafer (1966). In Common Predicament: Social Psychology of Intergroup Conflict and Cooperation (Boston: Houghton-Mifflin).

Sidanius, Jim, and Felicia Pratto (2011) "Social Dominance Theory.” In Paul A. M. Van Lange, Arie W. Kruglanski, and E. Tory Higgins (eds.), Handbook of Theories of Social Psychology, Volume 2 (London: Sage): 418-38.

Snow, David A., E. Burke Rochford, Jr., Steven K. Worden and Robert D. Benford (1986) "Frame Alignment Processes, Micromobilization, and Movement Participation," American Sociological Review 51(4): 464-81.

Spector, Paul E., and Valentina Bruk-Lee (2008) “Conflict, Health, and Well-Being.” In Carsten K.W. De Dreu and Michele J. Gelfand (eds.), The Psychology of Conflict and Conflict Management in Organizations (New York: Lawrence Erlbaum Associates): 267-88. 
Tajfel, Henry and John Turner (1979) “An Integrative Theory of Intergroup Conflict.” In William G. Austin and Stephen Worchel (eds.), The Social Psychology of Intergroup Relations (Monterey, CA: Brooks-Cole): 33-47.

Thompson, Leigh L., and Brian J. Lucas (2014) "Judgmental Biases in Conflict Resolution and How to Overcome Them.” In Peter T. Coleman, Morton Deutsch, and Eric C. Marcus (eds.), The Handbook of Conflict Resolution: Theory and Practice, 3rd ed. (San Francisco: Jossey-Bass): 255-82.

Thompson, Paul, and David McHugh (2009) Work Organisations: A Critical Approach, 4th ed. (London: Palgrave Macmillan).

Terry, Deborah J., and Catherine E. Amiot (2008) "Social Identification Processes, Conflict, and Fairness Concerns in Intergroup Mergers.” In Carsten K.W. De Dreu and Michele J. Gelfand (eds.), The Psychology of Conflict and Conflict Management in Organizations (New York: Lawrence Erlbaum Associates): 385-411.

Tinsley, Catherine H. (2001) "How Negotiators Get to Yes: Predicting the Constellation of Conflict Management Strategies Used Across Cultures,” Journal of Applied Psychology 86(4): 583-93.

Tversky, Amos, and Daniel Kahneman (1974) "Judgment Under Uncertainty: Heuristics and Biases,” Science 185(4157): 1124-31.

Ury, William L., Jeanne M. Brett, and Stephen B. Goldberg (1989) Getting Disputes Resolved: Designing Systems to Cut the Costs of Conflict (San Francisco: Jossey-Bass).

van Meurs, Nathalie, and Helen Spencer-Oatey (2007) "Multidisciplinary Perspectives on Intercultural Conflict: The 'Bermuda Triangle' of Conflict, Culture and Communication.” In Helga Kotthoff and Helen Spencer-Oatey (eds.), Handbook of Intercultural Communication (Berlin: de Gruyter): 99-122.

Walsh, James P. (1995) "Managerial and Organizational Cognition: Notes from a Trip Down Memory Lane,” Organization Science 6(3): 280-321.

Walton, Richard E., and Robert B. McKersie (1965) A Behavioral Theory of Labor Negotiations (New York: McGraw-Hill).

Weber, Libby, and Kyle Mayer (2014) "Transaction Cost Economics and the Cognitive Perspective: Investigating the Sources and Governance of Interpretive Uncertainty,” Academy of Management Review 39(3): 344-63.

Zariski, Archie (2010) “A Theory Matrix for Mediators,” Negotiation Journal 26(2): 203-35. 
Table 1

A Three-Factor Framework on the Sources of Conflict

\begin{tabular}{ll}
\hline Factor & Definition and Dimensions \\
\hline Structural & The nature of the relationship between the interests or goals of two or more \\
& people or organizations \\
& - Self-interested exchange with accessible alternatives (egoist) \\
& - Lasting interdependence with mutual gains structure (unitarist) \\
& - Lasting interdependence with mixed-motive structure (pluralist) \\
& - Lasting interdependence with win-lose structure (antagonistic) \\
& Individual or group mental maps, assessments, or framing of a situation \\
Cognitive & - Cognitive frames and preferences \\
& - (In- and Out-) group perceptions \\
& (Mis)communication \\
& Individual affective reactions to situations and other people \\
Psychogenic & Emotions and moods \\
\hline
\end{tabular}

